\title{
Spin transition in a four-coordinate iron oxide
}

\section{AUTHOR(S):}

Kawakami, Takateru; Tsujimoto, Yoshihiro; Kageyama, Hiroshi; Chen, Xing-Qiu; Fu, C. L.; Tassel, Cedric; Kitada, Atsushi; ... Nasu, S.; Podloucky, R.; Takano, Mikio.

\section{CITATION:}

Kawakami, Takateru ...[et al]. Spin transition in a four-coordinate iron oxide. Nature Chemistry 2009, 1: 371-376

\section{ISSUE DATE:}

2009-07

URL:

http://hdl.handle.net/2433/85013

\section{RIGHT:}

c 2009 Nature Publishing Group. 許諾条件により本文は2010-01-01に公 開.; This is not the published version. Please cite only the published version.; この論文は出版社版でありません。引用の際には出版社版を ご確認ご利用ください。 


\section{Spin transition in a four-coordinate iron oxide}

T. Kawakami ${ }^{1}$, Y. Tsujimoto ${ }^{2}$, H. Kageyama ${ }^{2}$, Xing-Qiu Chen ${ }^{3}$, C. L. Fu ${ }^{3}$, C. Tassel ${ }^{2}$, A.

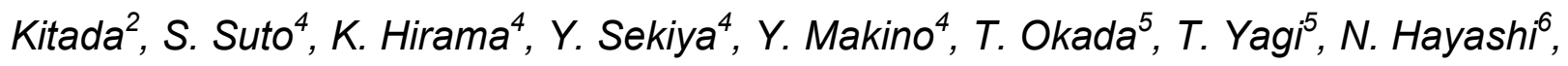
K. Yoshimura ${ }^{2}$, S. Nasu ${ }^{7}$, R. Podloucky ${ }^{8}$, M. Takano ${ }^{9}$

${ }^{1}$ Institute of Quantum Science, Nihon University, Chiyoda, Tokyo 101-8308, Japan.

${ }^{2}$ Department of Chemistry, Graduate School of Science, Kyoto University, Sakyo, Kyoto 6068502, Japan.

${ }^{3}$ Materials Science and Technology Division, Oak Ridge National Laboratory, Oak Ridge, Tennessee 37831, USA.

${ }^{4}$ Graduate School of Quantum Science and Technology, Nihon University, Chiyoda, Tokyo 1018308, Japan.

${ }^{5}$ Institute for Solid State Physics, University of Tokyo, Kashiwa, Chiba 277-8581, Japan.

${ }^{6}$ Graduate School of Human and Environmental Studies, Kyoto University, Sakyo, Kyoto 6068501, Japan.

${ }^{7}$ Institute for Chemical Research, Kyoto University, Uji, Kyoto 611-0011, Japan

${ }^{8}$ Institute of Physical Chemistry, University of Vienna, Sensengasse 8/7, Wien, Austria

${ }^{9}$ Institute for Integrated Cell-Materials Science, Kyoto University, Yoshida Ushinomita-cho, Sakyo, Kyoto 606-0801, Japan. 
Spin transition has attracted interests of researchers in various fields since early 1930's, with thousands of materials being realized including minerals and biomolecules. However, so far the metal centers in which it occurs have been almost always octahedral sixcoordinate $3 d^{4-7}$ metals, such as $\mathrm{Fe}$ (II). A five-coordination is only rarely seen. Here we report that $\mathrm{SrFe}^{2+} \mathrm{O}_{2}$, featuring a four-fold square-planar coordination, exhibits a high-spin $(S=2)$ to intermediate-spin $(S=1)$ transition on pressurization. A transition from antiferromagnetic insulator to ferromagnetic metal transition takes place at the same time. The ferromagnetic $S=1$ state is found to be a half metal due to the inception of halfoccupied spin-down $\left(d_{x y}, d_{y z}\right)$ states. These results highlight the square-planar coordinated iron oxides as a new class of magnetic/electric materials.

Since the early 1930's the phenomenon of spin transition has attracted researchers of various fields, finding thousands of materials including minerals and biomolecules. In spite of all these efforts up to now, the metal centers, which undergo the spin transition, are nearly exclusively in an octahedral six-fold coordination such as Fe(II). A five-fold coordination is only rarely seen. Here we report that $\mathrm{SrFe}^{2+} \mathrm{O}_{2}$, featuring a four-fold square-planar coordination, exhibits a high-spin $(S=2)$ to intermediate-spin $(S=1)$ transition on pressurization. This transition is accompanied by a transition from antiferromagnetic insulating to a ferromagnetic so-called half-metallic states: only half of the spin-down $\left(d_{x y}, d_{y z}\right)$ are filled. These results highlight square-planar coordinated iron oxides as new class of magnetic/electric materials.

Spin transition, or spin crossover, generally occurs in compounds of octahedrally coordinated $3 d$ transition metal ions with $d^{4}, d^{5}, d^{6}$ and $d^{7}$ electronic configurations, and they are driven by the competition between the intra-atomic exchange energy and the crystal field energy. The former stabilizes a high-spin state, a spin state with a maximum spin multiplicity as for the free ion, while the latter stabilizes a low-spin state where the electrons occupy low energy orbitals only at the expense of increasing the exchange energy ${ }^{1}$. This transition, which can be induced by external perturbations such as heating ${ }^{2,3}$, pressurization ${ }^{4-8}$, lightning 9 , magnetic field $^{10}$, chemical substitution ${ }^{11}$, and gas adsorption ${ }^{12}$, were found in a wide range of materials like oxides $\left(\mathrm{FeBO}_{3}, \mathrm{CaFeO}_{3}\right)^{4}, 5,7,{ }^{10}$, metal-organic complexes ${ }^{1}$, porous materials ${ }^{12}$, 
supramolecular systems $^{3}$ as well as in human hemoglobin ${ }^{13}$. Some of these materials have found promising applications as sensors, or displaying and recording devices. Recent discoveries of the spin state transition in magnesiowüstite $(\mathrm{Mg}, \mathrm{Fe}) \mathrm{O}^{4,5}$ and silicate perovskite $(\mathrm{Mg}, \mathrm{Fe})(\mathrm{Si}, \mathrm{Al}) \mathrm{O}_{3}{ }^{7}$ have provided renewed understanding on the seismic wave heterogeneity in Earth's lower mantle. Among the ions that exhibit spin state transition phenomena, the largest number of cases is found for the $d^{6}$ electronic configuration, of which divalent iron(II) represents the majority. The transition has been observed mostly between the high-spin $(S=2)$ and the low-spin $(S=0)$ states.

The crystal field energy depends on the particular geometrical arrangement of both metal ion and ligands, as well as on the metal-ligand distance. It also depends on properties such as substantial deviations from octahedral symmetry, packing effects in crystal lattices, the thermal contraction inherent to crystalline solids, and cooperative interactions. For example, it is known that a transition involving an intermediate spin state $\left(S=1\right.$ for $d^{6}$ and $S=3 / 2$ for $\left.d^{5}\right)$ is feasible for five-fold coordinated compounds such as $\mathrm{TbBaCo}_{2} \mathrm{O}_{5}$ with a pryramidal coordination ${ }^{14}$ and the $\left[\mathrm{Fe}\left(\mathrm{P}_{4}\right) \mathrm{Br}\right] \mathrm{BPh}_{4} \cdot \mathrm{CH}_{2} \mathrm{Cl}_{2}$ complex ( $\mathrm{P}_{4}$ : hexapenyl-1,4,7,10-tetraphosphadecane, Ph: phenyl) with a trigonal-bypyramidal coordination ${ }^{15}$. To the authors' knowledge, however, up to now no spin transition is reported for compounds with a four or lesser coordinated metal ion.

A recently synthesized iron(II) oxide $\mathrm{SrFeO}_{2}$, as prepared by the hydride reduction of $\mathrm{SrFeO}_{3}$ at low temperatures, unprecedentedly has a square planar coordination of the high-spin iron(II) ion ${ }^{15,16}$. As shown in Fig. 1d, this material is built from two-dimensional $\mathrm{FeO}_{2}$ layers intervened by $\mathrm{Sr}$ atoms, isomorphic with the so-called infinite layer structure as first found for $(\mathrm{Ca}, \mathrm{Sr}) \mathrm{CuO}_{2}$. In spite of an apparent two-dimensional crystal structure, it undergoes an antiferromagnetic order at a remarkably high temperature (Néel temperature: $T_{\mathrm{N}}=473 \mathrm{~K}$ ) into a $G$-type spin structure, which is due to the strong hybridization between $\mathrm{Fe} d_{x-y}^{2}$ and $\mathrm{O} p_{\sigma}$ orbitals as evidenced by a very low isomer shift in the Mössbauer spectrum.

Here we present investigations of pressurization on the spin and electronic state in $\mathrm{SrFeO}_{2}$ by ${ }^{57} \mathrm{Fe}$ Mössbauer spectroscopy, electrical resistance, X-ray diffraction as well as by firstprinciples calculations. We found that $\mathrm{SrFeO}_{2}$ is the first material with a four-fold coordinated metal ion which shows a spin state transition. The high-spin to intermediate-spin transition is accompanied by an insulator-to-metal transition as well as an antiferromagnetic-to-ferromagnetic transition.

Typical high-pressure ${ }^{57} \mathrm{Fe}$ Mössbauer spectra of $\mathrm{SrFeO}_{2}$ at room temperature are shown in 
Fig. 2a (see Supplementary Fig. 1 for details). Below $30 \mathrm{GPa}$, no significant change was observed within the experimental uncertainties. The hyperfine field $\left(H_{\mathrm{hf}}\right)$ at $25 \mathrm{GPa}$, for example, is $42 \mathrm{~T}$, which is nearly identical with that at ambient pressure ${ }^{15}$, indicating that the divalent iron ion remains in the high-spin state $(S=2)$, and that $T_{\mathrm{N}}$ remains high. The gradual and linear decrease in the isomer shift (IS) with pressure (see Supplementary Fig. 2) is in accordance with the trend observed in many iron compounds under high pressure ${ }^{1,8}$ and can be interpreted in terms of gradually increased hybridization of $\mathrm{Fe} 3 d$ orbitals with $\mathrm{O} 2 p$ orbitals of the oxygen neighbors.

A drastic change, however, arose in the Mössbauer spectrum at a pressure of $38 \mathrm{GPa}$. While it also consists of well-defined six peaks indicating the presence of magnetic order at ambient temperature, $H_{\mathrm{hf}}$ of $18 \mathrm{~T}$ is nearly a half only of that for the low-pressure phase, implying the occurrence of a spin-state transition into an intermediate spin $(S=1)$ state. This spectrum feature remains up to the maximum pressure of $70 \mathrm{GPa}$ as applied in this study. In order to confirm the spin state transition, in other words to exclude the possibility that the reduction in $H_{\mathrm{hf}}$ is merely due to the reduction of $T_{\mathrm{N}}$, we cooled down the sample to $7 \mathrm{~K}$. The corresponding spectrum has an only slightly increased value of $H_{\mathrm{hf}}$ of $19 \mathrm{~T}$, providing firm evidence that the iron ions in the high-pressure phase are in the intermediate spin state. The well-developed six-line pattern in the intermediate spin state indicates that the magnetic order temperature is still far above room temperature. Subsequently, Mössbauer spectroscopy of the high-pressure phase was conducted under a magnetic field, for which the incident $\gamma$-ray was set parallel to the applied magnetic field. As shown in Fig. 2 and in Supplementary Fig. 3, the intensities of the 2nd and 5th lines $(\Delta m=0)$ showed a remarkable decrease with increasing magnetic field and disappeared at $3 \mathrm{~T}$. We thus conclude that the high-pressure phase is a ferromagnet. The quadrupole shift $\left(Q S=\mathrm{S}_{1}-\mathrm{S}_{2}\right)$ changed suddenly from $1.53 \mathrm{mms}^{-1}(25 \mathrm{GPa})$ to $0.70 \mathrm{mms}^{-1}(38 \mathrm{GPa})$ after the transition. Both the initial and the new spectra coexist in the pressure range 27-37 GPa due to a pressure gradient within the sample. Yet, the analysis of the relative spectrum weight and the pressure gradient allowed a rough estimation of the critical pressure $P_{\mathrm{c}}$ of $33 \pm 3 \mathrm{GPa}$.

To examine a possibility of structural change on the spin transition, we carried out in situ Xray powder diffraction experiments in a diamond anvil cell at pressures up to $43 \mathrm{GPa}$. The diffraction data recorded (see Supplementary Fig. 4) are of high quality with a good signal-tonoise ratio, allowing accurate determination of the lattice parameters. All the peaks of the 
diffraction pattern at each pressure could be assigned to tetragonal infinite-layer structure, and no additional reflections were detected within the resolution of the experiment. Though the measured range of the diffraction angles is not sufficient to make a final conclusion, it is likely that the transition is isostructural. At pressures below the spin transition, the pressure dependence of the volume can be fitted by the third order Birch-Murnaghan equation of state with the bulk modulus $K=126 \mathrm{GPa}$, which is slightly smaller than that estimated for the fully oxidized perovskite $\mathrm{SrFeO}_{3}(K=146 \mathrm{GPa})^{17}$. As displayed in Fig. 1a, the unit cell parameters $a$ and $c$ decrease smoothly with the application of pressure, but exhibit a significant drop at $33 \pm 1 \mathrm{GPa}$, which agrees well with the pressure $P_{\mathrm{c}}$ as estimated from the Mössbauer study. Therefore, this anomaly might be associated with the claimed spin transition. A volume reduction of $\sim 3 \%$ (Fig. 1b) is comparable with those for other compounds that exhibit high-spin to low-spin transitions ${ }^{1}$.

First-principles calculations based on the hybrid density functional theory (PBE0) method ${ }^{18,}$ ${ }^{19}$ were carried out to examine the pressure dependence of the crystal and electronic structures. The antiferromagnetic $S=2$ state is the ground state at atmospheric pressure and remains stable up to $P_{\mathrm{c}}$, where the abrupt transition to the ferromagnetic $S=1$ state occurs (Fig. 2b), verifying the experimental observations. The calculated $P_{\mathrm{c}}$ of $53 \mathrm{GPa}$ is, however, rather high in comparison with the experimental $P_{\mathrm{c}}$ of $33 \mathrm{GPa}$. As shown in Fig. 2c, the calculated spin moment decreases from $3.5 \mu_{\mathrm{B}}$ to $1.8 \mu_{\mathrm{B}}$ ( $\mu_{\mathrm{B}}$ : bohr magneton) between the antiferromagnetic and ferromagnetic states, which is consistent with the change in $H_{\mathrm{hf}}$ from $42 \mathrm{~T}$ to $19 \mathrm{~T}$. With respect to the $a$ axis, both the theory and experiment indicated a drop of $\sim 2 \%$, but concerning the $c$ axis, the sign of change is opposite between the theory and experiment. However, in terms of the unit cell volume at $P_{\mathrm{c}}$, the discrepancy between theory and experiment differs only by $3 \AA^{3}$ (or $6 \%$ ).

To understand the nature of the spin transition, we first focus on the antiferromagnetic $S=2$ state. The local density-of-states of the Fe ion at atmospheric pressure is shown in Fig. 4a. Consistent with the results of previous "LDA+U" calculations ${ }^{20,21}$, the lone spin-down electron is found to occupy the $d_{z}{ }^{2}$ orbital. The double occupation of $d_{z}^{2}$ allows a considerable reduction of the intra-atomic Coulomb repulsion ${ }^{20,21}$. The $\sigma$-bond formed between $\mathrm{Fe} d_{x-y}^{2}{ }^{2}$ and $\mathrm{O} p_{x, y}$ is split into the bonding and antibonding states by $\sim 7 \mathrm{eV}$ owing to a strong in-layer Fe $d$ and $\mathrm{O} p$ hybridization. For pressures less than $P_{c}$, both the bonding and antibonding states in the spin-up channel are occupied. In the antiferromagnetic $S=2$ state, the $d^{6}$ state has the electronic configuration of $\left(d_{z}^{2}\right)^{2}\left(d_{x z}, d_{y z}\right)^{2}\left(d_{x y}\right)^{1}\left(d_{x-y}^{2}\right)^{2}$. 
We find that the spin transition is attributed to the strong in-layer hybridization between Fe $d_{x-y}^{2}-\mathrm{O} p_{\sigma}$ bonding states leading to electronic instability toward the depopulation of $d_{x-y}^{2}-\mathrm{O}$ $p_{\sigma}$ antibonding states. The depopulation of the Fe $d_{x-y}^{2}{ }^{2}$ antibonding states can be seen in Fig. $4 \mathrm{~b}$ showing the local deinsity-of-states of Fe for the ferromagnetic $S=1$ state. The Fe $d$ electrons are increased to $d^{6.5}$ in total with the electronic configuration of $\left(d_{z}^{2}\right)^{1}\left(d_{x z}, d_{y z}\right)^{2}\left(d_{x y}\right)^{1}\left(d_{x-y}^{2}{ }^{2}\right)^{0.25}$ for the spin-up channel and $\left(d_{z}^{2}\right)^{1}\left(d_{x z}, d_{y z}\right)^{1}\left(d_{x y}\right)^{0}\left(d_{x-y}^{2}\right)^{2} 0.25$ for the spin-down channel. Another point to be noted here is that the ferromagnetic $S=1$ state is half-metallic. In the antiferromagnetic $S=2$ state, the band gap decreases considerably with increasing pressure as shown in Fig. 3a, and then drops to naught at $P_{c}$.

The spin moment of the Fe $d_{x-y}^{2}{ }^{2}$ bonding states is quenched due to the enhanced in-layer bonding between Fe $d$ and $\mathrm{O} p$. The double occupation of $d_{z}^{2}$ is maintained in the ferromagnetic $S=1$ state just after the transition, but becomes energetically unfavorable due to the compression of the interlayer spacing. Therefore, the charge transfer from spin-down $d_{z}^{2}$ to spin-down $d_{x y}$ has to occur, while the spin-down $\left(d_{x z}, d_{y z}\right)$ states remain half-occupied (Fig. 4c). It follows that, as the pressure reaches $P \geq 70 \mathrm{GPa}$, the spin-down states of $d_{x y}$ and $d_{z}^{2}$ also become half-occupied in the ferromagnetic $S=1$ state $\left(d_{z}^{2}\right)^{0.5}\left(d_{x z}, d_{y z}\right)^{1}\left(d_{x y}\right)^{0.5}\left(d_{x-y}^{2}\right)^{0.25}$ for the spin-down states, resulting in an increase of density-of-states at the Fermi level.

We conducted electrical resistance measurement on non-sintered powder sample under pressure, as summarized in Fig. 3. At ambient and low pressures, $\mathrm{SrFeO}_{2}$ is a nonconductor or a semiconductor with an out-of-range resistance. The electrical resistance at room temperature became measurable above $17 \mathrm{GPa}$. Across the spin state transition, a drastic decrease of resistance occurs from kiloohm at $27 \mathrm{GPa}$ to ohm at $37 \mathrm{GPa}$, and to centiohm at $50 \mathrm{GPa}$. The temperature dependence of the electrical resistance $\Delta R / \Delta T$ becomes smaller, and finally its sign changes from negative to positive at $\sim 60 \mathrm{GPa}$. Although $\Delta R / \Delta T$ turns negative at lower temperatures, this is possibly due to Anderson localization and the use of the non-sintered powder sample. In addition, the resistance measurements suffered from the pressure distribution in the sample as in the Mössbauer experiments, which must render the insulator-metal transition at $P_{\mathrm{c}}$ obscured to some extent. It is likely that the intermediate spin state is a metal as theoretically suggested.

Comparing with the ferromagnetic $S=1$ state, the antiferromagnetic $S=1$ state is much less stable above $P_{\mathrm{c}}$. An examination of its electronic structure of the antiferromagnetic $S=1$ state 
(Fig. 4d) shows that, in addition to having a $d_{z}^{2}$ electron in the spin-down channel, the second spin-down electron actually prefers to occupy the $d_{x y}$ orbital. The antiferromagnetic $S=1$ state remains as an insulator. The sudden increase in the energy from antiferromagnetic $S=2$ to antiferromagnetic $S=1$ is due to the increase of Coulomb repulsion resulting from the double occupation of $d_{x y}$. We have also examined the possibility of spin transition induced by phonon instability at various pressures. More specifically, we considered the effects of Jahn-Teller lattice distortion and breathing phonon mode. The former could induce a spin state of $S=1$, while the latter could result in a coexistence of the $S=1$ and $S=2$ states due to a charge disproportionation reaction. However, we find experimentally and theoretically that $\mathrm{SrFeO}_{2}$ is stable against a cooperative oxygen displacement in either Jahn-Teller lattice distortion or breathing phonon mode in both antiferromagnetic and ferromagnetic states at all pressures. Therefore, the spin transition in $\mathrm{SrFeO}_{2}$ is not due to these phonon instabilities.

The spin state transitions in $3 d$ ions are routinely interpreted as due to the transfer of one or two electrons between $d$ orbitals of opposite spin channels. The observed $S=2$ to $S=1$ transition in $\mathrm{SrFeO}_{2}$ is distinctly different from the previously reported examples in two aspects: it involves the transfer of non-integer $d$ electrons and the $p \rightarrow d$ charge transfer (see Supplementary Table 1).

A range of transition-metal oxides $^{23-26}$ has now been found to exhibit half-metallic ferromagnetism - these are limited mostly to manganese and chromium oxides $\left(\mathrm{La}_{1.3} \mathrm{Sr}_{0.7} \mathrm{Mn}_{2} \mathrm{O}_{7}\right.$, $\mathrm{Tl}_{2} \mathrm{Mn}_{2} \mathrm{O}_{7}, \mathrm{CrO}_{2}$ etc.). The double-perovskite oxide $\mathrm{Sr}_{2} \mathrm{FeMoO}_{6}$ is known as iron-based halfmetallic ferromagnet, but it contains molybdenum which is rare in the earth crust. Since iron is the most abundant transition-metal, the discovery of half-metallic ferromagnetism in $\mathrm{SrFeO}_{2}$ containing only iron is important in view of future application in industry related to magnetism and spin electronics, although $P_{\mathrm{c}}$ is still too high. Recent studies ${ }^{27-29}$ have demonstrated that the square planar coordination around iron in $\mathrm{SrFeO}_{2}$ is not a mere fortuity but could be widely and universally available including serial spin-ladders $\mathrm{Sr}_{n+1} \mathrm{Fe}_{n} \mathrm{O}_{2 n+1}(n=$ integer $)$, such as $n=2$ $\mathrm{Sr}_{3} \mathrm{Fe}_{2} \mathrm{O}_{5}$. One can also distort a square planar $\mathrm{FeO}_{4}$ unit toward a tetrahedron as in $\mathrm{CaFeO}_{2}$. Although $P_{\mathrm{c}}$ in $\mathrm{SrFeO}_{2}$ is yet still too high, it is desirable to reduce $P_{\mathrm{c}}$ in those related iron oxides while keeping the transition temperature far above room temperature.

Metallization of the $\mathrm{FeO}_{2}$ sheet would provide us a hope that further pressurization could lead to superconductivity in analogy with the layered cuprates. It is also interesting to compare the present compound with a recently discovered iron oxypnictide superconductors ${ }^{30}$ since in 
both cases the iron atoms form two-dimensional square lattice and are coordinated by four anions. Furthermore, preliminary calculations at higher pressures suggest a transition from the $S=1$ state to a 2nd intermediate spin $(S=1 / 2)$ state before it finally falls onto the $S=0$ state. It is interesting to check these points experimentally.

\section{METHODS}

The powder sample of $\mathrm{SrFeO}_{2}$ was synthesized by the reducing reaction with $\mathrm{CaH}_{2}$ of a slightly oxygen deficient perovskite $\mathrm{SrFeO}_{2.875}$. The precursor $\mathrm{SrFeO}_{2.875}$ was prepared by a high-temperature ceramic method from $\mathrm{SrCO}_{3}(99.99 \%)$ and $\mathrm{Fe}_{2} \mathrm{O}_{3}(99.99 \%) . \mathrm{SrFeO}_{2.875}$ and a two-molar excess of $\mathrm{CaH}_{2}$ were mixed, finely ground in an Ar-filled drybox, sealed in an evacuated Pyrex tube, and reacted at $553 \mathrm{~K}$ for two days. The residual $\mathrm{CaH}_{2}$ and the $\mathrm{CaO}$ byproduct were removed from $\mathrm{SrFeO}_{2}$ by washing them out with a $\mathrm{NH}_{4} \mathrm{Cl}$ /methanol solution. For details, please refer to the literature ${ }^{15}$.

The high-pressure ${ }^{57} \mathrm{Fe}$ Mössbauer measurements were performed up to $70 \mathrm{GPa}$ using a Bassett-type diamond-anvil cell ${ }^{31}$. The ${ }^{57} \mathrm{Fe}$-enriched $\mathrm{SrFeO}_{2}$ powder and small ruby chips were enclosed in the hole of a Re gasket. Two types of pressure-transmitting media, a 4:1 methanol:ethanol solution and Daphne7373, were used. A point $\gamma$-ray source of ${ }^{57} \mathrm{Co}$ in a Rh matrix of $370 \mathrm{MBq}$ and another one of $925 \mathrm{MBq}$ having active areas of $1 \mathrm{~mm}$ and $4 \mathrm{~mm}$ in diameter, respectively, were used. The pressure was determined by means of ruby fluorescence manometry. To estimate the pressure distribution along the sample, several ruby chips were placed inside the hole at different distances from the center of the hole. It was found that the pressure gradient at the sample was not more than $8 \mathrm{GPa}$ at maximal pressures. The $\gamma$-ray source could not be narrowed more because the high-pressure Mössbauer study allows only a limited sample and a long exposure time as long as a week was required to accumulate fairly reasonable spectral statistics. The magnetic field was produced by a superconducting solenoid operating in the persistent mode up to 3 T. ${ }^{57} \mathrm{Fe}$ Mössbauer experiments under the external magnetic field were carried out with the magnetic field applied along the $\gamma$-ray propagation direction. The velocity scale of the spectrum was relative to $\alpha$-Fe at room temperature. Electrical resistance was measured with a standard dc four-probe method between $5 \mathrm{~K}$ and $300 \mathrm{~K}$ up to $67 \mathrm{GPa}$. Fine alumina powder and $\mathrm{NaCl}$ were pressed onto the gasket surface for electrical insulation and pressure-transmitting medium, respectively, on which sample powder and Pt electrodes were 
placed together. Applied pressure was measured by means of fluorescence manometry on ruby chips placed around the sample. In this electrical resistance measurement the sectional area and the distance between probes were about $60 \mu \mathrm{m} \times 50 \mu \mathrm{m}$ and $50 \mu \mathrm{m}$, respectively.

Powder X-ray diffraction profiles at high pressures up to $43 \mathrm{GPa}$ were recorded using Mo- $K_{\alpha}$ radiation from a $5.4 \mathrm{~kW}$ Rigaku rotating anode generator equipped with a $100 \mu \mathrm{m}$ collimator. Powder sample of $\mathrm{SrFeO}_{2}$ was loaded in a $300 \mu \mathrm{m}$ hole of preindented stainless-steel gasket of the diamond-anvil cell. A 4:1 methanol:ethanol mixture was used as the pressure-transmitting medium. The shift of ruby fluorescence was used for the determination of pressure. To estimate the pressure distribution along the sample, several ruby chips were placed inside the hole at different distances from the center of the hole. It was found that the pressure gradient at the sample was not more than $0.5 \mathrm{GPa}$ at maximal pressures. The diffracted X-ray was collected with an image plate. More details of the experimental setup are reported elsewhere ${ }^{32}$. Four diffraction peaks, $110,011,020$, and 121 were used to calculate the cell parameters.

The first-principles calculations were performed using the Vienna ab initio Simulation Package (VASP) ${ }^{33}$ with the ion-electron interaction described by the projector augmented wave potential (PAW) ${ }^{34}$ (see Supplementary Information for details). We employed the hybrid density functional theory (PBE0) method ${ }^{18,35}$, as implemented in $\mathrm{VASP}^{19,36}$, to treat the localized $\mathrm{Fe}^{2+}$ states in $\mathrm{SrFeO}_{2}$. The exchange energy in PBE0 mixes the energies of exact Hartree-Fock (HF) exchange and PBE exchange from the local density functional approximation ${ }^{19,36}$. The longrange part of the HF exchange interaction is included in PBE0. The weight of HF exchange in the mixing is general less than $25 \%$. We find that the weights between $10 \%-20 \%$ of $\mathrm{HF}$ exchange provide the most consistent results and describe the experimental findings reasonably well for $\mathrm{SrFeO}_{2}$. The results reported in this paper were done by using $15 \% \mathrm{HF}$ with $85 \%$ of $\mathrm{PBE}$ for the exchange energy. We find that the spin transition pressure from the antiferromagnetic $(S$ $=2$ ) state to the ferromagnetic state is insensitive to the weights of mixing. It is the stable ranges of the $S=2$ and $S=1$ states within the ferromagnetic ordering that shows an increased sensitivity to the weights of HF mixing in PBE0.

In our calculations, the semicore states (the $3 p$ and $4 s$ states of $\mathrm{Sr}$ and the $3 p$ state of $\mathrm{Fe}$ ) were treated as valence states. An energy cutoff of $500 \mathrm{eV}$ was chosen for the plane wave expansion. Full relaxation of atomic positions and lattice parameters at each pressure was achieved by minimizing forces and stress tensor components. Forces were minimized down to 
$10^{-5} \mathrm{eV} \AA^{-1}$ and the difference in the total energies between two successive electronic iterations was required to be less than $10^{-7} \mathrm{eV}$. The angular momentum projected local density of states and magnetic moments of Fe were calculated within a sphere (i.e., the Wigner-Seitz radius) of $1.164 \AA$.

\section{References}

[1] Gütlich, P. \& Goodwin, H. A. Spin crossover in transition metal compounds I-III, vol. 233235, Springer-Verlag Heidelberg (2004).

[2] Kröber, J., Codjovi, E., Kahn, O., Croliére, F. \& Jay, C. A spin transition system with a thermal hysteresis at room temperature. J. Am. Chem. Soc. 115, 9810-9811 (1993).

[3] Real, J. A., Andés, E., Muñoz, M. C., Julve, M., Granier, T., Bousseksou, A. \& Varret, F. Spin crossoer in a catenane supramolecular system. Science 267, 265-267 (1995).

[4] Badro, J., Fiquet, G., Guyot, F., Ruett, J.-P., Struzhkin, V. V., Vankó, G. \& Monaco, G. Iron partitioning in earth's mantle: toward a deep lower mantle discontinuity. Science 300, 789791 (2003).

[5] Lin, J.-F., Struzhkin, V. V., Jacobsen, S. D., Hu, M. Y., Chow, P., Kung, J., Liu, H., Mao, H.K. \& Hernley, R. J. Spin transition of iron in magnesiowüstite in the earth's lower mantle. Nature 436, 377-380 (2005).

[6] Rueff, J.-P., Kao, C.-C., Struzhkin, V. V., Bardo, J., Shu, J., Hemley, R. J. \& Mao, H. K. Pressure-induced high-spin to low-spin transition in FeS evidenced by X-ray emission spectroscopy. Phys. Rev. Lett. 82, 3284-3287 (1999).

[7] Li, J., Struzhkin, V. V., Mao, H.-K., Shu, J., Jemley, R. J., Fei, Y., Mysen, B., Dera, P., Prakapenka, V. \& Shen, G. Electronic spin state of iron in lower mantle perovskite. Proc. Natl. Acad. Sci. U.S.A. 101, 14027-14030 (2004).

[8] Takano, M., Nasu, S., Abe, T., Yamamoto, K., Endo, S., Takeda, Y. \& Goodenough, J. B. Pressure-induced high-spin to low-spin transition in $\mathrm{CaFeO}_{3}$. Phys. Rev. Lett. 67, 3267-3270 (1991).

[9] Decurtins, S., Gütlich, P., Köhler, C. P., Spiering, H. \& Hauser, A. Light-induced excited spin state trapping in a transition-metal complex: the hexa-1-propyltetrazole-iron (II) tetrafluoroborate spin-crossover system. Chem. Phys. Lett. 105, 1-4 (1984).

[10] Qi, Y., Müller, E. W., Spiering, H. \& Gütlich, P. The effect of a magnetic field on the high- 
spin $\leftrightarrow$ low-spin transition in $\left[\mathrm{Fe}(\text { phen })_{2}(\mathrm{NCS})_{2}\right]$. Chem. Phys. Lett. 101, 505-505 (1983).

[11] Ikeue, T., Ohgo, Y., Yamaguchi, T., Takahashi, M., Takeda, M. \& Nakamura, M. Saddleshaped six-coordinate iron(III) porphyrin complexes showing a novel spin crossover between $S=1 / 2$ and $S=3 / 2$ spin state. Angew. Chem Int. Ed. 40, 2617-2620 (2001).

[12] Halder, G. J., Kepert, C. J., Moubaraki, B., Murray, K. S. \& Cashion, J. D. Guest-dependent spin crossover in a nanoporous molecular framework material. Science 298, 1762-1765 (2002).

[13] Nakano, N., Nakano, K. \& Tasaki, A. A magnetic study of aciditic ferric hemoglobin. Biochem. Biophys. Acta. 251, 301-313 (1971).

[14] Moritomo, Y., Akimoto, T., Takeo, M., Machida, A., Nishibori, E., Takata, M., Sakata, M., Ohoyama, K. \& Nakamura, A. Metal-insulator transition induced by a spin-state transition in $\mathrm{TbBaCo}_{2} \mathrm{O}_{5+\delta}(\delta=0.5)$, Phys. Rev. B. 61, R13325-13328 (2000).

[15] Tsujimoto, Y., Tassel, C., Hayashi, N., Watanabe, T., Kageyama, H., Yoshimura, K., Takano, M., Ceretti, M., Ritter, C. \& Paulus, W. Infinite-layer iron oxide with a squareplanar coordination. Nature 450, 1062-1065 (2007).

[16] Köhler, J. Square-planar coordinated iron in the layered oxoferrate (II) $\mathrm{SrFeO}_{2}$. Angew. Chem. Int. Ed. 47, 4470-4472 (2008).

[17] Kawakami, T., Nasu, S., Kuzushita, K., Sasaki, T., Morimoto, S., Yamada, T., Endo, S., Kawasaki, S. \& Takano, M. High-Pressure Mössbauer and X-Ray Powder Diffraction Studies of $\mathrm{SrFeO}_{3}$.J. Phys. Soc. Jpn. 72, 33-36 (2003).

[18] Perdew, M., Ernzerhof, M \& Bruke, A. Rationale for mixing exact exchange with density functional approximations. J. Chem. Phys. 105, 9982-9985 (1996).

[19] Paier, J., Marsman, M., Hummer, K., Kresse, G., Gerbert, I. C \& Ángyán, J. G. Screened hybrid density functionals applied to solids. J. Chem. Phys. 124, 154709 (2006).

[20] Xiang, H. J., Wei, Su-Huai \& Whangbo, M.-H. Origin of the Structural and Magnetic Anomalies of the Layered Compound $\mathrm{SrFeO}_{2}$ : A Density Functional Investigation. Phys. Rev. Lett. 100, 167207 (2008).

[21] Pruneda, J. M., Inigquez, J., Canadell, E., Kageyama, H. \& Takano, M. Understanding the Unique Structural and Electronic Properties of $\mathrm{SrFeO}_{2}$. Phys. Rev. B 78, 115101 (2008).

[22] Hwang, H. Y. \& Cheong, S.-W. Enhanced intergrain tunneling magnetoresistance in halfmetallic $\mathrm{CrO}_{2}$ films. Science 278, $1607-1609$ (1997). 
[23] Kimura, T., Tomioka, Y., Kuwahara, H., Asamitsu, A., Tamura, M. \& Tokura, Y. Interplane Tunneling Magnetoresistance in a layered manganite crystal. Science 274, 1698-1701 (1996).

[24] Okimoto, Y., Katsufuji, T., Ishikawa, T, Urushibara, A., Arima, T. \& Tokura, Y. Anomalous variation of optical spectra with spin polarization in double-exchange ferromagnet: $\mathrm{La}_{1-x} \mathrm{Sr}_{x} \mathrm{MnO}_{3}$. Phys. Rev. Lett. 75, 109-112 (1995).

[25] Shimakawa, Y., Kubo, Y. \& Manako, T. Giant magnetoresistance in $\mathrm{Tl}_{2} \mathrm{Mn}_{2} \mathrm{O}_{7}$ with the pyrochlore structure. Nature 379, 53-55 (1996).

[26] Park, J.-H, Vescova, E., Kim, H.-J., Kwon, C., Ramesh, R. \& Ventakesan, T. Direct evidence for a half-matallic ferromagnet. Nature 794, 794-796 (1998).

[27] Kageyama, H., Watanabe, T., Tsujimoto, Y., Kitada, A., Sumida, Y., Kanamori, K., Yoshimura, K., Hayashi, N., Muranaka, S., Takano, M., Ceretti, M., Paulus, W., Ritter, C. \& André, G. Spin-ladder iron oxide $\mathrm{Sr}_{3} \mathrm{Fe}_{2} \mathrm{O}_{5}$. Angew. Chem. Int. Ed. 47, 5740-5745 (2008).

[28] Tassel, C., Watanabe, T., Tsujimoto, Y., Hayashi, N., Kitada, A., Sumida, Y., Yamamoto, T., Kageyama, H., Takano, M. \& Yoshimura, K. Stability of the infinite layer structure with iron square planar coordination. J. Am. Chem. Soc. 130, 3764-3765 (2008).

[29] Tassel, C., Pruneda, J. M., Hayashi, N., Watanabe, T., Kitada, A., Tsujimoto, Y., Kageyama, H., Yoshimura, K., Takano, M., Nishi, M., Ohoyama, K., Mizumaki, M., Kawamura, N., Íñiguez, J. \& Canadell, E. $\mathrm{CaFeO}_{2}$ : a new type of layered structure with iron in a distorted square planar coordination. J. Am. Chem. Soc. 130, 214410-214419 (2008).

[30] Kamihara, Y.; Watanabe, T.; Hirano, M.; Hosono, H. Iron-based layered superconductor $\mathrm{La}\left[\mathrm{O}_{1-x} \mathrm{~F}_{x}\right] \mathrm{FeAs}(x=0.05-0.12)$ with $T_{\mathrm{c}}=26$ K. J. Am. Chem. Soc. 130, 3296-3297 (2008).

[31] Bassett, W. A., Takahashi, T. \& Stook, P. W. X-Ray Diffraction and Optical Observations on Crystalline Solids up to 300 kbar. Rev. Sci. Instrum. 38, 37-42 (1967).

[32] Arora, A. K., Yagi, T., Miyajima, N., Mary, T. A. Amorphization and decomposition of scandium molybdate at high pressure. J. Appl. Phys. 97, 013508 (2005).

[33] Kresse, G. \& Furthmuller, J. Efficiency of ab-initio total energy calculations for metals and semiconductors using a plane-wave basis set. Comput. Mater. Sci. 6, 15-50 (1996).

[34] Kresse, G. \& Joubert, D. From ultrasoft pseudopotentials to the projector augmented-wave method. Phys. Rev. B 59, 1758-1775 (1999).

[35] Perdew J. P., Burke K., Ernzerhof M., Generalized Gradient Approximation Made Simple, Phys. Rev. Lett. 77, 3865-3868 (1996). 
[36] Paier, J., Hirschl, R., Marsman, M. \& Kresse, G. The Perdew-Burke-Ernzerhof exchangecorrelation functional applied to the G2-1 test set using a plane-wave basis set. J. Chem. Phys. 122, $234102(2005)$.

Acknowledgements This work was supported by Science Research on Priority Areas "Novel States of Matter Induced by Frustration" (No. 19052004) and also partly by another one (No. 17105002) from the Ministry of Education, Culture, Sports, Science and Technology of Japan. Research at Oak Ridge National Laboratory was sponsored by the Division of Materials Sciences and Engineering, U. S. Department of Energy under contract with UT-Battelle, LLC. This research used resources of the National Energy Research Computing Center, which is supported by the Office of Science of the U.S. Department of Energy under Contract No. DE-AC0205CH11231. This work was supported by the University of Vienna through the University Focus Research Area Materials Science "Multi-scale Simulations of Materials Properties and Processes in Materials". Correspondence and requests for materials should be addressed to H.K. (kage@kuchem.kyoto-u.ac.jp)

\section{Author contributions}

H.K designed and coordinated the overall study seeking advice from M.T (experiment) and C.L.F (theory). N.H conceived high-pressure Mössbauer study, while X-Q.C conceived theoretical studies. Y.T synthesized the material. T.K, S.S, K.H, Y.S and Y.M conducted highpressure Mössbauer and electrical registivity experiments, and T.K analyzed the data with the help of M.T and S.N. Y.T, C.T, A.K, T.O and T.Y performed high-pressure X-ray diffraction experiments, and Y.T and C.T analyzed the data. X-Q.C, C.L.F and R.P performed the theoretical work and analysis. H.K and T.K co-wrote the experimental part and C.L.F and X-Q.C 
co-wrote the theoretical part with comments from R.P. All contributed to the discussion of the results.

\section{Figure Legends}
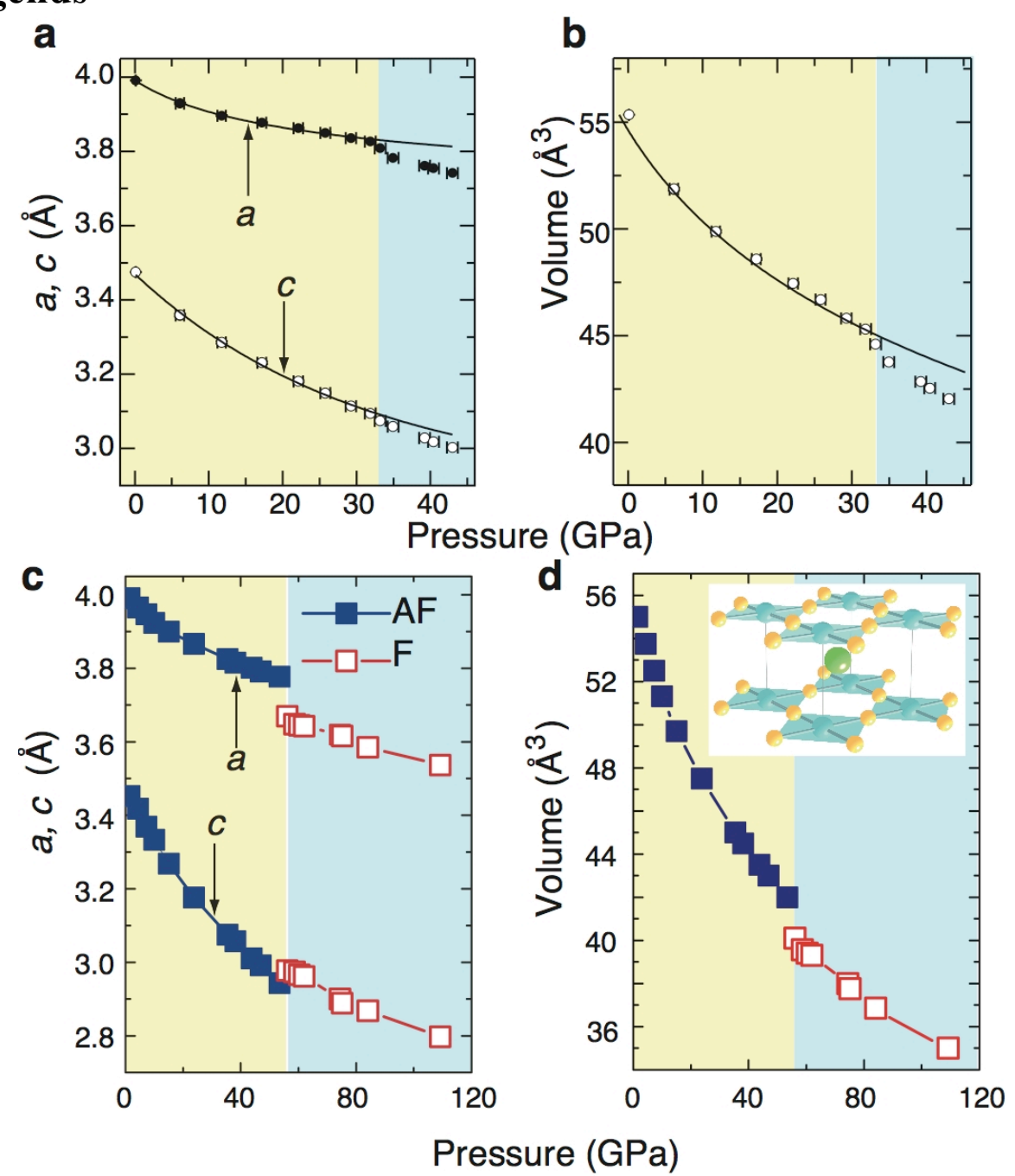

Fig. 1. Lattice anomaly at the spin state transition. The lattice parameters $a, c$ (a) and the volume $V(\mathbf{b})$ as a function of pressure. The errors bars are s.d. determined by the least-squares fitting, and are within the size of the symbols. The solid lines in a are the guide to the eyes and that in $\mathbf{b}$ is the fit to the third order Brich-Murnagam in the low-pressure phase. c, $\mathbf{d}$ Calculated cell parameters and volume for the $S=2$ antiferromagnetic (AF) state and the $S=1$ ferromagnetic (F) state. Inset of $\mathbf{d}$ is the crystal structure of $\mathrm{SrFeO}_{2}$, where blue, green and yellow balls represent $\mathrm{Fe}, \mathrm{Sr}$ and $\mathrm{O}$ atoms, respectively. 

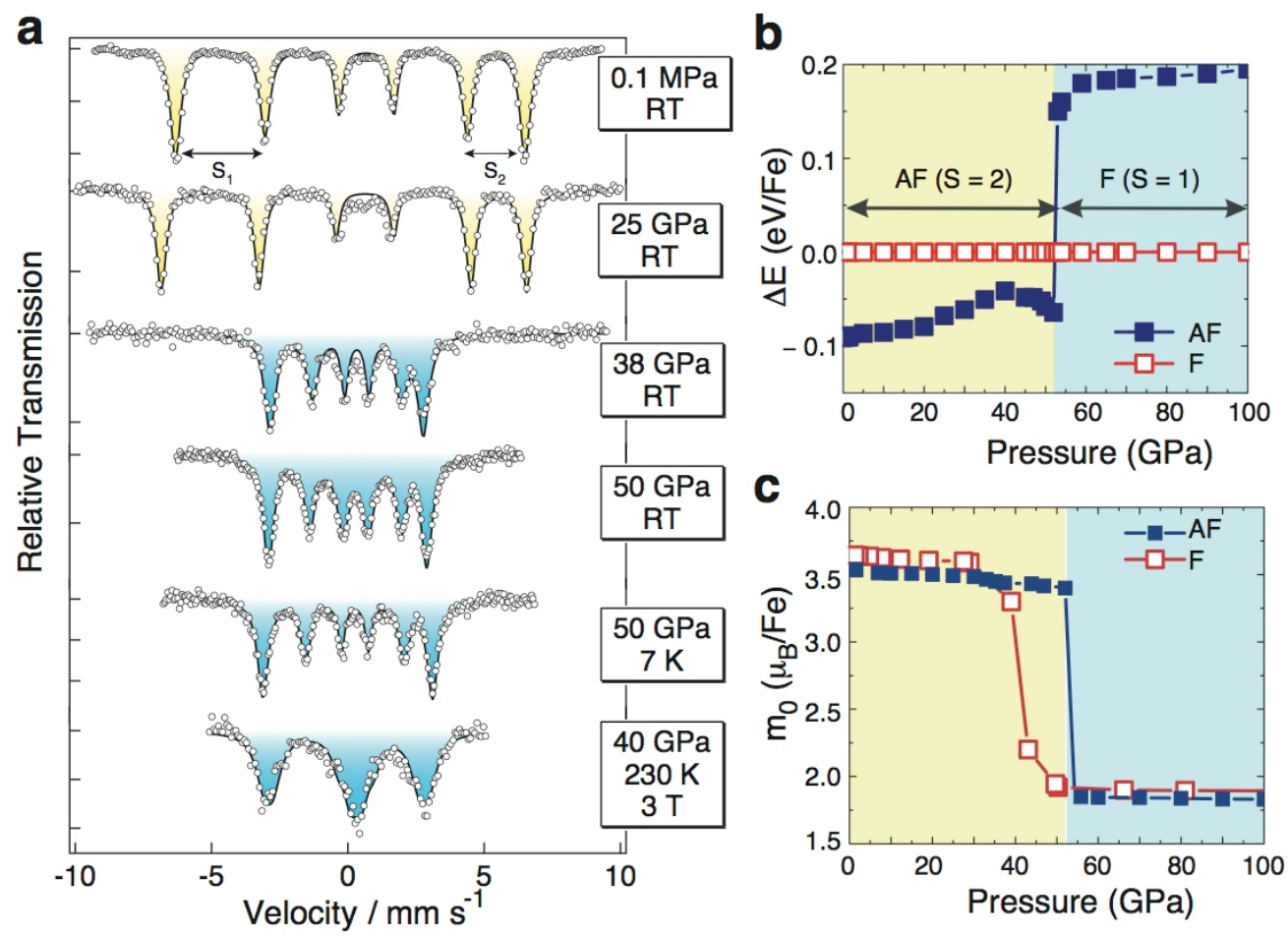

Fig. 2. High-spin-to-intermediate-spin transition in $\mathrm{SrFeO}_{2}$ under high pressure. a, Typical high-pressure ${ }^{57} \mathrm{Fe}$ Mössbauer spectra obtained from $\mathrm{SrFeO}_{2}$ (see Supporting Fig. 1 for detail). The yellow spectra correspond to the $S=2$ state, while the blue to the $S=1$ state. The solid lines represent the fitted curves. Velocity scale is relative to $\alpha$-Fe at room temperature. $\mathbf{b}$, Calculated energy difference $(\Delta \mathrm{E})$ between the $S=2$ antiferromagnetic (AF) state and the $S=1$ ferromagnetic (F) state. c, Calculated spin moment $\left(m_{0}\right)$ for the $S=2$ antiferromagnetic (AF) state and the $S=1$ ferromagnetic (F) state. 

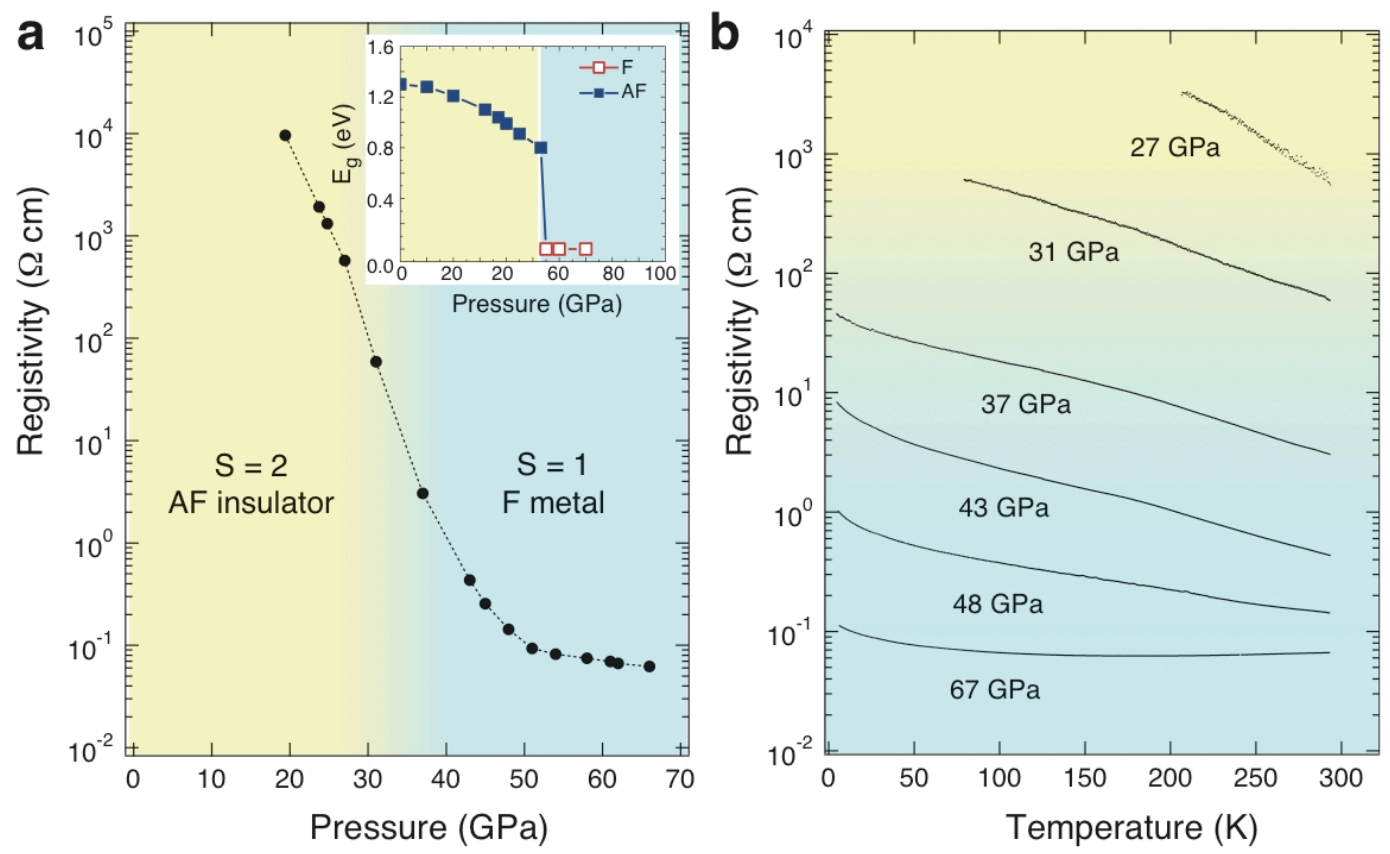

Fig. 3. Insulator metal transition at the spin transition. a, Room temperature electrical resistivity of $\mathrm{SrFeO}_{2}$, demonstrating an insulating state for the low-pressure phase $\left(P<P_{\mathrm{c}}\right)$ and a metallic state for the high-pressure phase $\left(P>P_{\mathrm{c}}\right)$. The inset represents the calculated energy gap for the $S=2$ antiferromagnetic (AF) state and the $S=1$ ferromagnetic (F) state, in consistency with the experimental results. b, Temperature dependence of the electrical resistivity under pressure. 

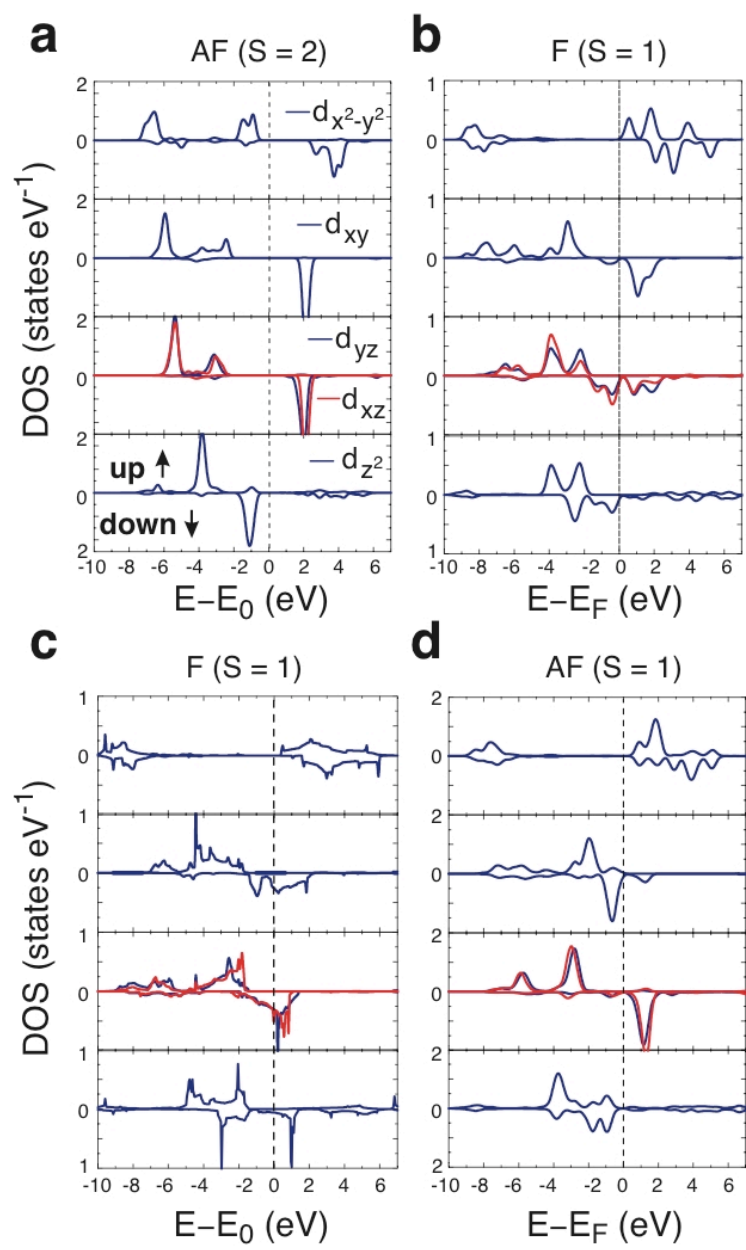

Fig. 4. Calculated local density-of-states of $\mathbf{F e}$ in $\mathrm{SrFeO}_{2}$. a, high-spin $(S=2)$ antiferromagnetic (AF) state at $0 \mathrm{GPa}$ (insulating); $\mathbf{b}$, intermediate-spin $(S=1)$ ferromagnetic (F) state at $53 \mathrm{GPa}$ (metallic); c, intermediate-spin $(S=1)$ ferromagnetic (F) state at $70 \mathrm{GPa}$ (metallic); d, unstable intermediate-spin $(S=1)$ antiferromagnetic (AF) state at $65 \mathrm{GPa}$ (insulating). In the $S=2$ antiferromagnetic state, the lone spin-down electron occupies the spindown $d_{z}{ }^{2}$ orbital. In the $S=1$ ferromagnetic state, the $d_{x-y}^{2}{ }^{2}$ antibonding states become unoccupied, which results in the charge transfer from $d_{x-y}^{2}$ to $d_{y z}$ (or $d_{x z}$ ). The $d_{z}^{2}$ orbital maintains its double occupation through the spin state transition at $53 \mathrm{GPa}$. The energy zero $\left(E_{0}\right)$ is set at the middle of the gap (a and $\mathbf{d}$ ) and at the Fermi level (b and $\mathbf{c}$ ). 